\title{
QUADRATIC POLYNOMIAL OF POWER SUMS AND ALTERNATING POWER SUMS
}

\author{
Leomarich F. Casinillo \\ Department of Mathematics and Physics, Visayas State University, Visca, Baybay City, Leyte, Philippines \\ Email : leomarich_casinillo@yahoo.com
}

\begin{abstract}
This study aims to construct quadratic polynomials in $\mathrm{n}$ for power sums and alternating power sums of consecutive positive integers. In addition, this paper evaluated the said quadratic polynomials under odd and even terms of the series.
\end{abstract}

Keywords: Quadratic polynomial, power sums, alternating power sums

\section{INTRODUCTION}

In number theory and combinatorics, power sum series and alternating power sum series of consecutive positive integers are widely investigated concepts, which remains intriguing and give much attention to research $[1,2,3,4,5,6]$. There are also some scientist and applied mathematicians uses the said series for mathematical real life applications [7,8]. Let $n$ and $m$ be a positive integer. Then, it is well known, among others, that the sum of the $p$-th power of the first $t$ positive integers

and

$$
S_{t}^{o}(1, n, p)=\sum_{j=1}^{t=2 n-1} j^{p}=1^{p}+2^{p}+3^{p}+\cdots+t^{p}
$$

$$
S_{t}^{e}(1, n, p)=\sum_{j=1}^{t=2 n} j^{p}=1^{p}+2^{p}+3^{p}+\cdots+t^{p}
$$

are closely connected to the Bernoulli polynomials $B_{p}(x)[9,10,11]$. In equation (1) and (2), the series has odd and even terms, respectively. The next series (3) and (4), represents as power sum of consecutive positive integer with $\mathrm{m}^{p}$ as the first term with odd and even terms, respectively. Indeed, here it is

$$
S_{t}^{o}(m, n, p)=\sum_{j=m}^{m+2 n-2} j^{p}=m^{p}+(m+1)^{p}+(m+2)^{p}+\cdots+(m+2 n-2)^{p}
$$

where $t \equiv 1(\bmod 2)$, and

$$
\begin{aligned}
& S_{t}^{e}(m, n, p) \\
& \quad=\sum_{j=m}^{m+2 n-1} j^{p}=m^{p}+(m+1)^{p}+(m+2)^{p}+\cdots+(m+2 n-1)^{p}
\end{aligned}
$$


where $t \equiv 0(\bmod 2)$. For the alternating power sum series of the first $t$ positive integers, we have the following equations for $m=1$,

$$
A_{t}^{o}(1, n, p)=\sum_{j=1}^{t=2 n-1} j^{p}=1^{p}-2^{p}+3^{p}-\cdots+t^{p}
$$

and also,

$$
A_{t}^{e}(1, n, p)=\sum_{j=1}^{t=2 n} j^{p}=1^{p}-2^{p}+3^{p}-\cdots-t^{p}
$$

In general, if $m$ is even, we have

$$
A_{t}^{o}(m, n, p)=\sum_{j=m}^{m+2 n-2}(-1)^{j} j^{p}=m^{p}-(m+1)^{p}+\cdots+(m+2 n-2)^{p}
$$

where $t=2 n-1$, and

$$
A_{t}^{e}(m, n, p)=\sum_{j=m}^{m+2 n-1}(-1)^{j} j^{p}=m^{p}-(m+1)^{p}+\cdots-(m+2 n-1)^{p}
$$

where $t=2 n$. Considering that $m$ is odd, then, we obtain

$$
A_{t}^{o}(m, n, p)=\sum_{j=m}^{m+2 n-2}(-1)^{j+1} j^{p}=m^{p}-(m+1)^{p}+\cdots+(m+2 n-2)^{p}
$$

where $t=2 n-1$, and lastly, we have

$$
A_{t}^{e}(m, n, p)=\sum_{j=m}^{m+2 n-1}(-1)^{j+1} j^{p}=m^{p}-(m+1)^{p}+\cdots-(m+2 n-1)^{p}
$$

where $t=2 n$. This paper investigates the construction process of quadratic polynomial in $n$ which gives the explicit formula for power sums and alternating power sums.

\section{RESULTS}

The following theorem is quick from equation (3).

Theorem 2.1. Let $n$ and $p$ be positive integers and let $t=2 n-1$. If $p=1$ and $n \geq 1$, or $p \geq 2$ and $n \leq 3$ then,

$$
\begin{gathered}
S_{t}^{o}(1, n, p)=\left[\frac{1}{2}\left(4^{p}+5^{p}-2^{p}-3^{p}\right)\right] n^{2}+\left\{\frac{1}{2}\left[5\left(2^{p}\right)+5\left(3^{p}\right)-3\left(4^{p}\right)-3\left(5^{p}\right)\right]\right\} n \\
+\left[5^{p}+4^{p}-2\left(3^{p}\right)-2\left(2^{p}\right)+1\right] .
\end{gathered}
$$


Proof: Let $t=2 n-1$. Suppose that $n$ and $p$ be positive integers. Then, we assume that $S_{t}^{o}(1, n, p)=a n^{2}+b n+c$, and $a=\alpha_{1}(p), b=\alpha_{2}(p)$ and $c=\alpha_{3}(p)$ are parameters to be estimated which are functions of positive integer $p$. Simulating the series $S_{t}^{o}(1, n, p)$ for $n \in$ $\{1,2,3\}$, then we obtain the following system of linear equations

$$
\left\{\begin{array}{c}
a+b+c=1 \\
4 a+2 b+c=1+2^{p}+3^{p} \\
9 a+3 b+c=1+2^{p}+3^{p}+4^{p}+5^{p}
\end{array}\right.
$$

Reducing the system of equations in (12) to triangular form, then we have

$$
\left\{\begin{array}{c}
a+b+c=1 \\
3 a+b=2^{p}+3^{p} \\
2 a=4^{p}+5^{p}-2^{p}-3^{p}
\end{array}\right.
$$

Applying the backward substitution in (13), it follows that

$$
\left\{\begin{array}{c}
a=\frac{1}{2}\left(4^{p}+5^{p}-2^{p}-3^{p}\right) \\
b=\frac{1}{2}\left[5\left(2^{p}\right)+5\left(3^{p}\right)-3\left(4^{p}\right)-3\left(5^{p}\right)\right] \\
c=5^{p}+4^{p}-2\left(3^{p}\right)-2\left(2^{p}\right)+1
\end{array}\right.
$$

Hence, this completes the proof of Theorem 1.

It is worth noting that Theorem 3 shows that $S_{t}^{o}(1, n, p)=a n^{2}+b n+c$ works only for $p=1$ and $n \geq 1$, or $p \geq 2$ and $n \leq 3$ whenever $t=2 n-1$. In Table 1, it illustrates some

\begin{tabular}{|c|c|c|}
\hline$p$ & $S_{t}^{o}(1, n, p)=a n^{2}+b n+c, t=2 n-1$ & Series \\
\hline 1 & $S_{t}^{o}(1, n, 1)=2 n^{2}-n$ & $1^{1}+2^{1}+3^{1}+\cdots+t^{1}$ \\
\hline 2 & $S_{t}^{o}(1, n, 2)=14 n^{2}-29 n+16$ & $1^{2}+2^{2}+3^{2}+\cdots+t^{2}$ \\
\hline 3 & $S_{t}^{o}(1, n, 3)=77 n^{2}-196 n+120$ & $1^{3}+2^{3}+3^{3}+\cdots+t^{3}$ \\
\hline 4 & $S_{t}^{o}(1, n, 4)=392 n^{2}-1079 n+688$ & $1^{4}+2^{4}+3^{4}+\cdots+t^{4}$ \\
\hline 5 & $S_{t}^{o}(1, n, 5)=1937 n^{2}-5536 n+3600$ & $1^{5}+2^{5}+3^{5}+\cdots+t^{5}$ \\
\hline 6 & $S_{t}^{o}(1, n, 6)=9464 n^{2}-27599 n+18136$ & $1^{6}+2^{6}+3^{6}+\cdots+t^{6}$ \\
\hline
\end{tabular}
quadratic polynomial in $n$ for $1 \leq p \leq 6$.

Table 1. Quadratic polynomials of sum series for $p \in\{1,2,3,4,5,6\}$ under odd terms.

The next result is immediate from the definition of the series $S_{t}^{o}(1, n, p)$ where $t \equiv$ $0(\bmod 2)$.

Theorem 2.2. Let $n$ and $p$ be positive integers and let $t=2 n$. If $p=1$ and $n \geq 1$, or $p \geq 2$ and $n \leq 3$ then,

$$
\begin{aligned}
S_{t}^{e}(1, n, p)=[ & \left.\frac{1}{2}\left(5^{p}+6^{p}-3^{p}-4^{p}\right)\right] n^{2}+\left\{\frac{1}{2}\left[5\left(3^{p}\right)+5\left(4^{p}\right)-3\left(5^{p}\right)-3\left(6^{p}\right)\right]\right\} n \\
& +\left[6^{p}+5^{p}-2\left(4^{p}\right)-2\left(3^{p}\right)+2^{p}+1\right] .
\end{aligned}
$$


Proof: Supposing $n$ and $p$ are positive integers and we let $t=2 n-1$. Again, we assume that $S_{t}^{e}(1, n, p)=a n^{2}+b n+c$, where $a=\beta_{1}(p), b=\beta_{2}(p)$ and $c=\beta_{3}(p)$ are parameters which are functions of positive integer $p$. So, we simulate the series $S_{t}^{e}(1, n, p)$ for $n \in\{1,2,3\}$, then we obtained the following system of linear equations

$$
\left\{\begin{array}{c}
a+b+c=1+2^{p} \\
4 a+2 b+c=1+2^{p}+3^{p}+4^{p} \\
9 a+3 b+c=1+2^{p}+3^{p}+4^{p}+5^{p}+6^{p}
\end{array}\right.
$$

By the method of elimination, the system (16) follows as

$$
\left\{\begin{array}{c}
a+b+c=1+2^{p} \\
3 a+b=3^{p}+4^{p} \\
2 a=5^{p}+6^{p}-3^{p}-4^{p}
\end{array}\right.
$$

So, by backward substitution in (17), it quickly follows that

$$
\left\{\begin{array}{c}
a=\frac{1}{2}\left(5^{p}+6^{p}-3^{p}-4^{p}\right) \\
b=\frac{1}{2}\left[5\left(3^{p}\right)+5\left(4^{p}\right)-3\left(5^{p}\right)-3\left(6^{p}\right)\right] \\
c=6^{p}+5^{p}-2\left(4^{p}\right)-2\left(3^{p}\right)+2^{p}+1
\end{array}\right.
$$

Hence, the proof of Theorem 2 is complete.

By simulation, Theorem 2 reveals that $S_{t}^{e}(1, n, p)=a n^{2}+b n+c$ is only applicable for $p=1$ and $n \geq 1$, or $p \geq 2$ and $n \leq 3$ whenever $t=2 n$. Table 2 illustrates some quadratic

\begin{tabular}{|c|c|c|}
\hline$p$ & $S_{t}^{e}(1, n, p)=a n^{2}+b n+c, t=2 n$ & Series \\
\hline 1 & $S_{t}^{e}(1, n, 1)=2 n^{2}+n$ & $1^{1}+2^{1}+3^{1}+\cdots+t^{1}$ \\
\hline 2 & $S_{t}^{e}(1, n, 2)=18 n^{2}-29 n+16$ & $1^{2}+2^{2}+3^{2}+\cdots+t^{2}$ \\
\hline 3 & $S_{t}^{e}(1, n, 3)=125 n^{2}-284 n+168$ & $1^{3}+2^{3}+3^{3}+\cdots+t^{3}$ \\
\hline 4 & $S_{t}^{e}(1, n, 4)=792 n^{2}-2039 n+1264$ & $1^{4}+2^{4}+3^{4}+\cdots+t^{4}$ \\
\hline 5 & $S_{t}^{e}(1, n, 5)=4817 n^{2}-13184 n+8400$ & $1^{5}+2^{5}+3^{5}+\cdots+t^{5}$ \\
\hline 6 & $S_{t}^{e}(1, n, 6)=28728 n^{2}-81359 n+52696$ & $1^{6}+2^{6}+3^{6}+\cdots+t^{6}$ \\
\hline
\end{tabular}
polynomial in $n$ for $1 \leq p \leq 6$

Table 2. Quadratic polynomials of sum series for $p \in\{1,2,3,4,5,6\}$ under even terms.

The following theorem shows the explicit formula of the series $A_{t}^{o}(1, n, p)$ whenever $t \equiv$ $1(\bmod 2)$. The series is positive for all positive integer $n$ and $p$.

Theorem 2.3. Let $n$ and $p$ be positive integers and let $t=2 n-1$. If $p \leq 2$ and $n \geq 1$, or $p \geq 3$ and $n \leq 3$ then,

$$
\begin{gathered}
A_{t}^{o}(1, n, p)=\left[\frac{1}{2}\left(5^{p}+2^{p}-4^{p}-3^{p}\right)\right] n^{2}+\left\{\frac{1}{2}\left[3\left(4^{p}\right)+5\left(3^{p}\right)-5\left(2^{p}\right)-3\left(5^{p}\right)\right]\right\} n \\
+\left[5^{p}+2\left(2^{p}\right)-4^{p}-2\left(3^{p}\right)+1\right]>0 .
\end{gathered}
$$


Proof: Since $t \equiv 1(\bmod 2)$, then, there are $\frac{t+1}{2}$ positive terms and $\frac{t-1}{2}$ negative terms in the series. Clearly, the sum of positive terms is greater than the absolute value of the sum of negative terms which follows that $A_{t}^{o}(1, n, p)>0$ for all positive integer $p$. Now, assume that $A_{t}^{o}(1, n, p)=a n^{2}+b n+c$, where $a=\gamma_{1}(p), b=\gamma_{2}(p)$ and $c=\gamma_{3}(p)$ are parameters which are function of positive integer $p$. Simulate the series $A_{t}^{o}(1, n, p)$ under $n \in\{1,2,3\}$, then we obtained the following system equations below

$$
\left\{\begin{array}{c}
a+b+c=1 \\
4 a+2 b+c=1-2^{p}+3^{p} \\
9 a+3 b+c=1-2^{p}+3^{p}-4^{p}+5^{p}
\end{array}\right.
$$

Applying the method of elimination in (20), we have,

$$
\left\{\begin{array}{c}
a+b+c=1 \\
3 a+b=-2^{p}+3^{p} \\
2 a=2^{p}-3^{p}-4^{p}+5^{p}
\end{array}\right.
$$

Finally, by backward substitution, it follows that

$$
\left\{\begin{array}{c}
a=\frac{1}{2}\left(5^{p}+2^{p}-4^{p}-3^{p}\right) \\
b=\frac{1}{2}\left[3\left(4^{p}\right)+5\left(3^{p}\right)-5\left(2^{p}\right)-3\left(5^{p}\right)\right] \\
c=5^{p}+2\left(2^{p}\right)-4^{p}-2\left(3^{p}\right)+1
\end{array}\right.
$$

Thus, substituting the solution to equation $A_{t}^{o}(1, n, p)=a n^{2}+b n+c$, then this completes the proof.

Theorem 3 reveals that $A_{t}^{o}(1, n, p)=a n^{2}+b n+c>0$ works only for $p \leq 2$ and $n \geq 1$, or $p \geq 3$ and $n \leq 3$ whenever $t=2 n-1$. Table 3 shows some quadratic polynomial in $n$ for $1 \leq p \leq 6$.

Table 3. Quadratic polynomials of alternating sign series for $p \in\{1,2,3,4,5,6\}$ under odd terms.

\begin{tabular}{|c|c|c|}
\hline $\boldsymbol{p}$ & $\boldsymbol{A}_{\boldsymbol{t}}^{\boldsymbol{o}}(\mathbf{1}, \boldsymbol{n}, \boldsymbol{p})=\boldsymbol{a \boldsymbol { n } ^ { 2 }}+\boldsymbol{b n}+\boldsymbol{c}, \boldsymbol{t}=\mathbf{2 n - 1}$ & Series \\
\hline 1 & $A_{t}^{o}(1, n, 1)=n$ & $1^{1}-2^{1}+3^{1}-\cdots+t^{1}$ \\
\hline 2 & $A_{t}^{o}(1, n, 2)=2 n^{2}-n$ & $1^{2}-2^{2}+3^{2}-\cdots+t^{2}$ \\
\hline 3 & $A_{t}^{o}(1, n, 3)=21 n^{2}-44 n+24$ & $1^{3}-2^{3}+3^{3}-\cdots+t^{3}$ \\
\hline 4 & $A_{t}^{o}(1, n, 4)=152 n^{2}-391 n+240$ & $1^{4}-2^{4}+3^{4}-\cdots+t^{4}$ \\
\hline 5 & $A_{t}^{o}(1, n, 5)=945 n^{2}-2624 n+1680$ & $1^{5}-2^{5}+3^{5}-\cdots+t^{5}$ \\
\hline 6 & $A_{t}^{o}(1, n, 5)=5432 n^{2}-15631 n+10200$ & $1^{6}-2^{6}+3^{6}-\cdots+t^{6}$ \\
\hline
\end{tabular}

The next Theorem provides the explicit formula of the series $A_{t}^{e}(1, n, p)$ whenever $t \equiv$ $0(\bmod 2)$. The series is strictly negative for all positive integer $n$ and $p$.

Theorem 2.4. Let $n$ and $p$ be positive integers and let $t=2 n$. If $p \leq 2$ and $n \geq 1$, or $p \geq 3$ and $n \leq 3$ then, 


$$
\begin{aligned}
A_{t}^{e}(1, n, p)= & {\left[\frac{1}{2}\left(5^{p}-6^{p}-3^{p}+4^{p}\right)\right] n^{2}+\left\{\frac{1}{2}\left[5\left(3^{p}\right)-5\left(4^{p}\right)-3\left(5^{p}\right)+3\left(6^{p}\right)\right]\right\} n } \\
& +\left[5^{p}-\left(6^{p}\right)-2\left(3^{p}\right)+2\left(4^{p}\right)-2^{p}+1\right]<0 .
\end{aligned}
$$

Proof: Let $n$ and $p$ be positive integers. Since $t \equiv 1(\bmod 2)$, then, there are $\frac{t}{2}$ positive terms and $\frac{t}{2}$ negative terms in the series. Now, the sum of positive terms is lesser than the absolute value of the sum of negative terms which clearly follows that $A_{t}^{o}(1, n, p)<0$ for all positive integer $p$. Again we assume that $A_{t}^{o}(1, n, p)=a n^{2}+b n+c$, where $a=\delta_{1}(p), b=\delta_{2}(p)$ and $c=\delta_{3}(p)$ are parameters which are functions of positive integer $p$. We now simulate the series $A_{t}^{e}(1, n, p)$ under $n \in\{1,2,3\}$, then we obtained the following system

$$
\left\{\begin{array}{c}
a+b+c=1-2^{p} \\
4 a+2 b+c=1-2^{p}+3^{p}-4^{p} \\
9 a+3 b+c=1-2^{p}+3^{p}-4^{p}+5^{p}-6^{p}
\end{array}\right.
$$

Applying the method of elimination, we have

$$
\left\{\begin{array}{c}
a+b+c=1-2^{p} \\
3 a+b=3^{p}-4^{p} \\
2 a=5^{p}-6^{p}-3^{p}+4^{p}
\end{array}\right.
$$

Finally, by backward substitution, it follows that

$$
\left\{\begin{array}{c}
a=\frac{1}{2}\left(5^{p}-6^{p}-3^{p}+4^{p}\right) \\
b=\frac{1}{2}\left[5\left(3^{p}\right)-5\left(4^{p}\right)-3\left(5^{p}\right)+3\left(6^{p}\right)\right] \\
c=5^{p}-\left(6^{p}\right)-2\left(3^{p}\right)+2\left(4^{p}\right)-2^{p}+1
\end{array}\right.
$$

Hence, the proof of Theorem 4 is complete.

The Theorem 4 above only covers $p \leq 2$ and $n \geq 1$, or $p \geq 3$ and $n \leq 3$ where $t=2 n$. It

\begin{tabular}{|c|c|c|}
\hline$p$ & $A_{t}^{e}(1, n, p)=a n^{2}+b n+c, t=2 n$ & Series \\
\hline 1 & $A_{t}^{e}(1, n, 1)=-n$ & $1^{1}-2^{1}+3^{1}-\cdots-t^{1}$ \\
\hline 2 & $A_{t}^{e}(1, n, 2)=-2 n^{2}-n$ & $1^{2}-2^{2}+3^{2}-\cdots-t^{2}$ \\
\hline 3 & $A_{t}^{e}(1, n, 3)=-27 n^{2}+44 n-24$ & $1^{3}-2^{3}+3^{3}-\cdots-t^{3}$ \\
\hline 4 & $A_{t}^{e}(1, n, 4)=-248 n^{2}+569 n-336$ & $1^{4}-2^{4}+3^{4}-\cdots-t^{4}$ \\
\hline 5 & $A_{t}^{e}(1, n, 5)=-1935 n^{2}+5024 n-3120$ & $1^{5}-2^{5}+3^{5}-\cdots-t^{5}$ \\
\hline 6 & $A_{t}^{e}(1, n, 6)=-13832 n^{2}+38129 n-24360$ & $1^{6}-2^{6}+3^{6}-\cdots-t^{6}$ \\
\hline
\end{tabular}
is worth noting that the theorem clearly implies that $A_{t}^{e}(1, n, p)<0$. In Table 4 , we consider $1 \leq p \leq 6$ as the power of the terms in the alternating sum series with even terms.

Table 4. Quadratic polynomials of alternating sign series for $p \in\{1,2,3,4,5,6\}$ under even terms. 


\section{CONCLUSION}

In this study, we had developed quadratic polynomials for power sums $S_{t}^{o}(1, n, p)$ and $S_{t}^{e}(1, n, p)$, and alternating power sums $A_{t}^{o}(1, n, p)$ and $A_{t}^{e}(1, n, p)$, where $n$ and $p$ are positive integers, and $t$ is the number of terms in the series. For power sums, the quadratic polynomials only works for $p=1$ and $n \geq 1$, or $p \geq 2$ and $n \leq 3$. On the other hand, for alternating power sums, the quadratic polynomials only works for $p \leq 2$ and $n \geq 1$, or $p \geq 3$ and $n \leq 3$. Additionally, it is concluded that $A_{t}^{o}(1, n, p)>0$ and $A_{t}^{e}(1, n, p)<0$.

\section{REFERENCES}

[1] Guy, R, "Sums of consecutive integers," Fibonacci Quarterly, vol. 20, pp. 36-38, 1982.

[2] LeVeque, W. J., "On representations as a sum of consecutive integers, " Canadian Journal of Mathematics, vol. 2, pp. 399-405, 1982.

[3] Merca, M., “An alternative to Faulhaber's formula," The American Mathematical Monthly, vol.122, no. 6, pp. 599-601, 2014.

[4] Howard F. T," "Sums of powers of integers via generating functions," Fibonacci Quarterly, vo. 34, pp. 244-256, 1996.

[5] Harman, G., "Sums of two squares in short intervals," Proceeding of London Mathematical Society, vol. 6, pp. 225-241, 1991.

[6] Bambah R. P. and Chowla S., "On numbers which can be express as a sum of two squares, ” Pro. Nat. Acad. Sci. India, vol. 13, pp. 101-103, 1947.

[7] Marin, M., "An evolutionary equation in thermoelasticity of dipolar bodies," Journal of Mathematical Physics, vol. 40, no. 3, pp. 1391-1399, 1999.

[8] Marin, M., "Contributions on Uniqueness in thermoelastodynamics on bodies with voids," Ciencias Mathematicas (Havana), vol. 16, no. 2, pp. 101-109, 1998.

[9] Bennett, M. A., "A super-elliptic equation involving alternating sums of powers," Publ. Math. Debrecen, vol. 79, pp. 317-324, 2011.

[10] Bazsó, A., "On alternating power sums of arithmetic progressions," Integral Transforms Spec. Funct, vol. 24, pp. 945-949, 2013.

[11] Bazsó, A., "Polynomial values of (alternating) power sums, "Acta Math. Hungary, vol. 146, pp. 202-219, 2015. 